ISSN 2693-2326

\title{
Improvement of the Pre-Analytical Phase of Nasopharyngeal Samples During the COVID 19 Pandemic: Experience of the Institute Pasteur De Côte d'Ivoire in 2020
}

Maxime Kouao DIANE, MD ${ }^{1,4 *}$, Claude Aimee DIAHA-KOUAME, PhD ${ }^{1,2}$, Herve A. KADJO, MD, PhD ${ }^{1,3}$, Stephane Kan KOUASSI, PhD ${ }^{1,6}$, Souleymane CISSE MD ${ }^{1,4}$, Marcelle MONEY, MD ${ }^{1,4}$, Edgard Valery ADJOGOUA, MD ${ }^{1,5}$ and Mireille DOSSO MD, $\mathrm{PhD}^{1}$

${ }^{1}$ Institut Pasteur de Cote d'Ivoire, Cote d'Ivoire

${ }^{2}$ Environment and Health Department

*Correspondence authors

${ }^{3}$ Respiratory Virus Unit

Maxime Kouao DIANE

MD

Biological Resource Center of Institut Pasteur de Côte

${ }^{4}$ Biological Resource Center

d'Ivoire

${ }^{5}$ Department of Epidemic Viruses

Submitted : 20 May 2021 ; Published : 5 Jun 2021

${ }^{6}$ Molecular biology platform

\begin{abstract}
The clinical laboratory is playing an increasingly important role in the patient-centered approach to the delivery of health services. Early detection of the importation of COVID-19 is a crucial challenge for all countries at risk of importation from areas of active transmission.
\end{abstract}

This article aims to share the experience of the IPCI in the optimum management of the pre-analytical phase related to diagnostic tests for COVID 19.

From the start of the pandemic the state and the IPCI made several organizational decisions to respond effectively to all trends of the COVID 19 epidemic in our country. Outsourcing of pre-analysis with creation of several sections: preparation of tube kits, aliquoting of samples from patients suspected of COVID19, reception, sorting and decontamination. Exemplary use of administrative and laboratory support staff.

Our findings should help establish urgent priorities for intensified preparedness and response support in specific African countries with moderate or high risk of COVID-19 importation and whose capacity to manage the health emergency is low relatively low.

Keywords: Abidjan, COVID-19, national reference laboratory, pre-analytical, riposte

\section{Introduction}

In late January 2020, the WHO declared the new Coronavirus disease 2019 (COVID-19) to be a public health emergency of international concern [1]. Africa, with a heterogeneity of economies, cultures and disease models, is fortunately the last continent to be affected by the pandemic [2]. Health systems in Africa are under strain and have minimal capacity to control the pandemic [3]. Early detection of the importation of COVID-19 is a crucial challenge for all countries at risk of importation from areas of active transmission [4].

The clinical laboratory is playing an increasingly important role in the patient-centered approach to the delivery of health services. Clinicians rely on accurate laboratory test results to correctly diagnose disease and guide therapy; it is estimated that over $70 \%$ of clinical decisions are based on information derived from laboratory test results [5].
Traditionally, the testing process has been divided into a pre-analytical phase, an analytical phase and a post-analytical phase. A series of published articles have drawn the attention of laboratory professionals to the pre and post-analytical phases, which currently appear to be more vulnerable to errors than the analytical phase [6-12]. The quality of the pre-analytical phase plays an essential role in obtaining reliable results, which promotes patient health, diagnosis and facilitates analysis of treatment effectiveness [13]. 


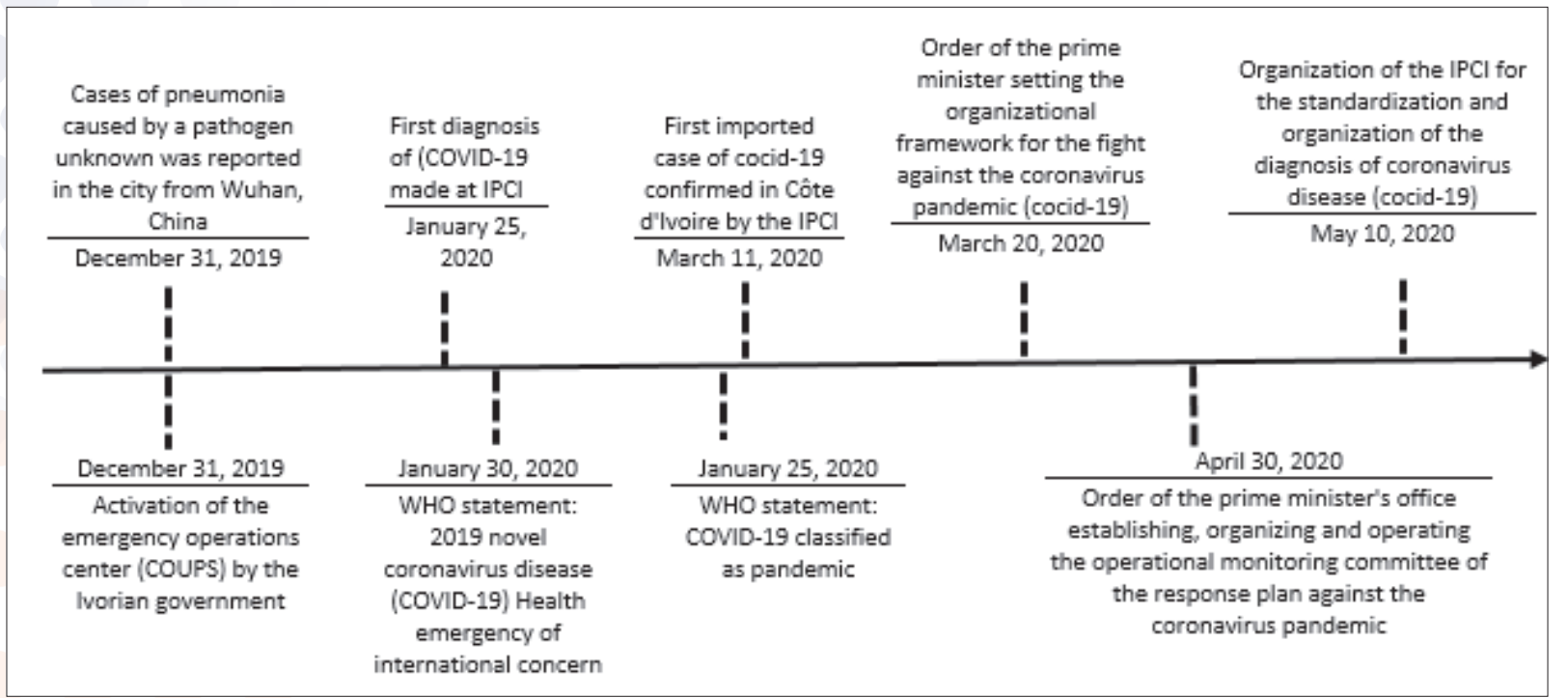

Figure 1: Chronological evolution of important events linked to the management of the COVID-19 pandemic in Côte d'Ivoire

Faced with the public health emergency and drawing on its successful experiences during the severe acute respiratory syndrome crisis in 2003, the H1N1 influenza pandemic in 2009, the Ebola epidemic crisis in 2014, Côte d'Ivoire addressed the management of the Coronavirus crisis by developing a national response plan (Figure 1) [14)]. With regard to laboratory tests, the respiratory virus laboratory of the Institut Pasteur de Côte d'Ivoire (IPCI) is the national reference laboratory (2006 Interministerial Order). It ensures the quality and standards of the tests according to the order of April 30, 2020 of the Prime Minister's office establishing, organizing and functioning of the operational monitoring committee of the response plan against the Coronavirus pandemic.

The first assessment of the diagnostic activities of the National Reference Laboratory (LNR) reported several gaps ranging from late delivery of results to the absence of results. An analysis of this situation revealed many irregularities, in particular, in the quality of the nasopharyngeal samples (sampling tube of various diameter not suitable or not usable by the laboratory, absence of viral transport media (VTM) in the tubes, the decontamination of the samples, the large number of microtube to prepare before the actual analysis, etc.) (unpublished data) as well as the technical organization of the pre-analytical phase including the labeling of the tube kits and aliquoting samples that increased the workload inside the laboratory.

This article aims to share the experience of the IPCI in the optimum management of the pre-analytical phase related to diagnostic tests for COVID 19.

\section{Materials and Methods}

This prospective work was carried out at the IPCI from January to October 2020 on the Adiopodoumé site. It was based, on the one hand, on the analysis of documents produced by the Ivorian government (response plan, decree and orders), of the Pasteur Institute of Côte d'Ivoire (decisions, memorandum, reports activities published or not, meeting minutes) and the activities of the established sections (monthly report). On the other hand, observations of the impact of the control of the pre-analytical phase of the samples on the detection process of SARS-COV2 by real-time PCR during the COVID-19 pandemic.

\section{Results}

Issue and management of epidemics

Epidemics are recurrent in Africa south of the Sahara. The health system regularly has to deal with epidemics. In 2019, Côte d'Ivoire had to manage the epidemic due to the Dengue virus (more than 6,000 suspected cases) which ended at the end of the year, a few weeks before the start of the pandemic to COVID-19.

Small and medium-scale epidemic: routine activity of the National Reference Laboratory.The National Institute of Public Hygiene (INHP) and the Pasteur Institute of Côte d'Ivoire (IPCI) are the primary actors in the management of epidemics. The INHP is responsible for collecting epidemiological data and samples throughout the country. The samples collected are then sent by him to the national reference laboratories located at the IPCI. The IPCI is responsible for confirming the diagnosis. Once the diagnosis is established, the results are given the INHP. It is this institute that is responsible for coordinating the response in terms of organization of prevention actions and treatment of cases by clinical services. 


\begin{tabular}{|l|l|l|l|l|}
\hline & Total samples & $\begin{array}{l}* \text { Average number of } \\
\text { samples per day }\end{array}$ & Total tube kits & $\begin{array}{l}\text { *Average number } \\
\text { of tube kits per day }\end{array}$ \\
\hline January & 7 & 0.2 & & \\
\hline February & 17 & 1 & & \\
\hline March & 1,284 & 41 & & \\
\hline April & 8,947 & 298 & & \\
\hline May & 19,100 & 637 & 16,000 & 516 \\
\hline June & 32,751 & 1,092 & 24,600 & 820 \\
\hline July & 30,346 & 979 & 37,200 & 1,200 \\
\hline August & 24,435 & 788 & 23,000 & 742 \\
\hline September & 32,735 & 1,091 & 37,200 & 1,240 \\
\hline October & 31,459 & 1,015 & 38,000 & 1,226 \\
\hline
\end{tabular}

Table 1: Monthly and daily distribution of the number of tube kits and sample for the diagnosis during the ten first month of covid-19 in Institut Pasteur de Côte d'Ivoire (IPCI) laboratory

* This average number per day was obtained by dividing the total monthly number by the number of days in the month.

At the start of the COVID 19 pandemic, the Ministry of Health and Public Hygiene activated its Public Health Emergency Operations Center (COUSP) with the establishment of a crisis committee in order to better manage the risk of contracting and spreading disease. It is within this framework that the IPCI has taken all the necessary measures to set up laboratory diagnosis with the help of the international network (World Health Organization, International Network of Institut Pasteur (RIIP) and Center for Diseases Control (CDC)). And the SARS COV2 RT PCR was available in Côte d'Ivoire as of January 25, 2020 [1]. From January to April 2020, within the IPCI, only the national reference laboratory for respiratory viruses assumed all stages of the diagnosis, from receipt of samples to dissemination of results. The first case of COVID 19 was diagnosed on March 11, 2020 by the National Reference Laboratory (CNR) for respiratory viruses of the IPCI. Table 1 shows that the respiratory virus lab received an average of 2 samples every 5 days in January and then went from about 1 sample per day in February to 298 samples per day in April thus saturating the capacity of laboratory staff to testing samples of steadily increasing COVID-19 cases alone. The stages of the diagnostic process consisted of the pre-analytical phase (reception - sorting and decontamination of samples, preparation of tubes, aliquoting of samples), of the analytical phase (inactivation, nucleic acid extraction, amplification) and post-analytical (recording of results and transmission). The Reference Laboratory in charge of respiratory viruses had to manage all these stages of the analysis on its own, while the number of samples from suspected cases was steadily increasing day by day.

Large-scale epidemic: definition of another concept and other strategies.

At the beginning of May 2020, the number of samples increased exponentially due to an outbreak of COVID-19 epidemics. There has been a transition from imported cases to community transmission of COVID-19 (appearance of indigenous cases) which has resulted in the overflow of the current strategy. This increase in the number of samples has forced the implementation of other strategies. We went from an average of 298 daily samples in April to over 639 daily samples in May (Table 1).

This situation has forced the management of the IPCI to redefine another concept and other strategies to contain this increase in the number of samples of up to 2000 samples per day according to the hypotheses of generalized community transmission of COVID-19.

\section{The big challenges were related to}

- Manual labeling of a large number of the tube kits for the COVID 19 diagnosis due to the increase in the number of infected people in the capital and throughout the territory. The corollaries were the increased workload in the laboratory, non-compliant and inadmissible samples.

- The diversity of collection tubes (various tubes with VTM were used) which arrived in laboratories authorized for the diagnosis of COVID-19 making the analytical phase of the laboratories difficult or even impossible.

- The inability of laboratories to adapt to this diversity of collection tubes and to implement corrective measures.

In view of these difficulties which punctuated the critical pre-analytical phases of the diagnostic process, the administration of the IPCI made the decision to improve the entire diagnostic process but especially this pre-analytical phase.

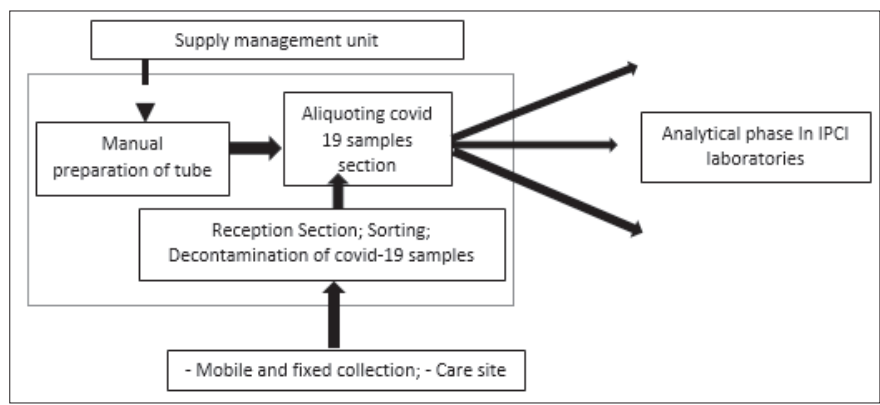

Figure 2: Box showing the different steps or processes of the pre-analytical phase set up at the Institut Pasteur de Côte d'Ivoire (IPCI) for the diagnosis of COVID 19 
Process implemented to improve the pre-analytical phase From the beginning of May 2020, the management of the IPCI made several organizational decisions to respond effectively to this new trend of the COVID 19 epidemic in our country.

1. Authorization of other laboratories to participate in the diagnosis.

- Within the IPCI, several decentralized teams of 5 to 6 people, biologists and laboratory technicians in other IPCI laboratories have been set up.

2. Deconcentrations and outsourcing of diagnostic tests of certain stages of the analysis process within the IPCI.

- The laboratories requisitioned within the IPCI were: the environment and health laboratory, the molecular biology platform (PFBM), the Biobank and the creation of new teams.

- Outside the IPCI: other laboratories were required to relocate the process of the COVID 19 diagnosis process (in Abidjan these are the laboratories of the Center for Diagnosis and Research on AIDS (CeDReS) and of RETROCI at the CHU of Treichville; in the interior of the country these are the laboratories of the regional hospitals).

3. Gradual automation of COVID analyzes.

4. Standardization of results reporting: a national results and sample management information system has been set up (bar code, tablet, transmission of results online).

5. Outsourcing of pre-analytics(figure 2) : he was created sections for the preparation of diagnostic tube kits and for aliquoting samples from patients suspected of COVID19. Aliquoting consisted of dispensing a sample into an already labeled $1.8 \mathrm{ml}$ cryotube. These samples in the 1.8 $\mathrm{ml}$ cryotube, which have become the primary samples, are immediately sent to the various laboratories for the analytical phase. This activity of preparing diagnostic tube kits has seen the significant contribution of administrative and laboratory support staff.

This organization of the diagnostic test set up by the IPCI aims to:

- $\quad$ Reduce the workload at analytical and post-analytical level

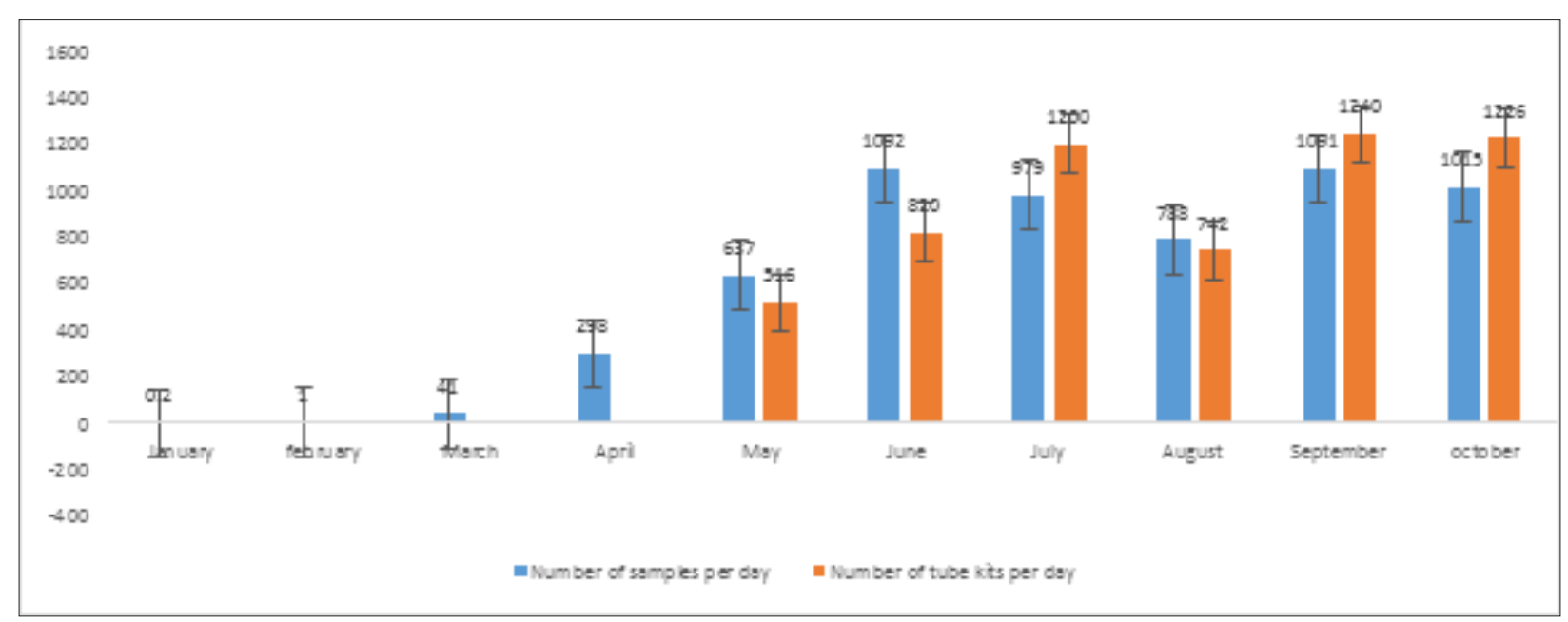

Figure 3: Distribution of the daily average of tube kits and samples during the ten first month of COVID-19 pandemic in laboratory of Institut Pasteur de Côte d'Ivoire (IPCI)

- Standardize laboratory techniques and conservation

- Reduce contamination

- Harmonize sample identification

Teams made up of support staff and temporary staff has been set up. They were trained in biosafety and biosafety in the laboratory. Achieving the objectives of the tube kit preparation teams involved setting up the tools: tube kit preparation sheets (type of tube, start number, end number) and a delivery of the tube kits to the aliquoting unit (delivery date, start number, end number, name of recipient, signature).

Regular meetings were made with the managers of the other teams intervening in the different processes of the pre-analytical and analytical phase (figure 2). These are the UGA, aliquoting section, reception section, sorting, decontamination of covid-19 samples and analysis laboratories. The purpose of these meetings is to adapt the number of tube kits to the needs. During these meetings several problem solving methods allowing to define and to bring solutions to the given problems were used. For this purpose certain tools (QQOQCP or "Quintilian Hexameter", brainstorming...) were used.

As part of this work, an analysis tube kit is defined as the set of microtubes used to diagnose a sample suspected of COVID 19 in the laboratory. The tube kit consisted of four microtubes: one $1.8 \mathrm{ml}$ cryotube, two eppendorf microtubes, one filter and column microtube. The number of microtubes has varied in the composition of the tube kit according to the development of the technique. This number ranged from four microtubes in the manual method of nucleic acid extraction to a microtube (a cryotube) in the automated method of extraction. The preparation of the tube kits consisted in writing on each microtube of the kit the serial number of the laboratory with an identification code of the recipient laboratory. (For example COV followed by a serial number for the respiratory virus laboratory COV 000001 and ECOV followed by a serial number for the chemistry and environmental microbiology laboratory ECOV 00001). 
Comments and challenges met

The sections put in place have made the process from nasopharyngeal sampling to the therapeutic management of COVID-19 cases and patient monitoring more fluid. During the reporting period from May to October, the tube kit preparation section produced 176,000 tube kits with an average production speed ranging from 516 tube kits per day in May to 1,200 tube kits per day in the months from July to October (Table 1). The aliquoting section processed 170,826 samples with an average of 637 samples in May to more than 1000 samples per day in September and October (Table 1). The goal during the establishment of these preparation sections of the covid-19 tubes and aliquoting kits was to improve the service delivery system of the COVID 19 laboratories in order to ensure the quality and standardization of the samples to move on to the analytical phase; reduce the time taken to return analysis results to a reasonable time by reducing the workload within the laboratory. The deadline for rendering results has been reduced from 7 days to 48 hours or even 24 hours if necessary. Figure 3 shows growth in COVID-19 sampling and diagnostic activities. The number of samples, which seemed to overwhelm diagnostic capacity, was quickly reduced and justifies the drastic reduction in the time taken to provide results and the working time of technical staff and laboratory biologists. The scenarios of the response plan predicted, in the extreme situation, a cumulative incidence of 30 cases per 100,000 inhabitants with an epidemic peak which could reach around 8,000 cases at the beginning of April 2020 (14).

These projections were effective so that from May 2020 the IPCI laboratories began to receive more than 1,000 daily samples. This positive effect was obtained through the combination of the various actions. The strategies put in place have enabled the two laboratories of the Institut Pasteur de Côte d'Ivoire (IPCI) to process more than $80 \%$ of the country's covid-19 samples. The drop in activity in August is explained by the use of a sample pooling technique which reduced the consumption of tube kits.

The introduction of so-called "open" automated systems in the diagnostic process has made it possible to accelerate the analytical phase of the laboratory and to minimize the risk of errors by harmonizing the identification of samples and identification of the tubes.

The standardization of laboratory techniques has made it possible to reduce costs and sample losses. Indeed, the repackaging of the samples taken in primary tubes of variable diameters and sizes which are sometimes difficult to use in the analytical phase, in $1.8 \mathrm{ml}$ cryotubes facilitates and standardizes this analysis in all laboratories.

The workload was reduced by the creation of aliquoting sections led by several groups of 4 people who took turns and strictly applying the biosecurity rules resulting from the training. This helped to reduce sample contamination. setting up the laboratory response plan and a coordination unit (ii) Decentralizing and deconcentrating the various phases of the diagnostic process (iii) Evaluating the diagnostic process (iv) pre-processing samples before taking them to external laboratories.

\section{Conclusion}

This study showed that the fight against the COVID-19 pandemic in Côte d'Ivoire required effective laboratory diagnosis. The IPCI has taken several steps necessary to improve the response to the COVID-19 pandemic. However, the march towards stopping the epidemic is not yet over. With more and more challenges to overcome, there are lessons to be learned every day. Our findings should help set urgent priorities for intensified support for preparedness and response to this or other outbreak in specific African countries with moderate or high risk of importing COVID-19 and whose capacity to manage the health emergency is relatively weak.

\section{Acknowledgments}

The authors would like to thank members of the Institute Pasteur of Côte d'Ivoire (IPCI) for assisting them in the management of data from IPCI database.

\section{Conflict of interest statement}

All authors have completed and submitted the International Committee of Medical Journal Editors form for disclosure of potential conflicts of interest. No potential conflicts of interest were disclosed.

\section{References}

1. WHO (2020). Statement on the second meeting of the International Health Regulations (2005) Emergency Committee regarding the outbreak of novel coronavirus (2019-nCoV) [Internet]. World Health Organization. [Cited 2020 Oct 16]. Available from: https://www.who.int/ news/item/30-01-2020-statement-on-the-second-meetingof-the-international-health-regulations-(2005)-emergency-committee-regarding-the-outbreak-of-novel-coronavirus-(2019-ncov).

2. Vanderpuye V, Elhassan MMA, Simonds H (2020). Preparedness for COVID-19 in the oncology community in Africa. Lancet Oncol, 21(5): 621-622.

3. WHO (2020). Laboratory testing for coronavirus disease († COVID-19) in suspected human cases: interim guidance, 19 March 2020. World Health Organization.

4. Gilbert M, Pullano G, Pinotti F, Valdano E, Poletto C, Boëlle P-Y, et al (2020). Preparedness and vulnerability of African countries against importations of COVID-19: a modelling study. Lancet Lond Engl, 14;395(10227): 871-7.

5. Datta P (2005). Resolving discordant specimens. Adv Adm $L a b, 60$.

6. Carraro P, Plebani M (2007). Errors in a stat laboratory: types and frequencies 10 years later. Clin Chem, 53(7):1338-1342.

The lessons learned for the management of this large-scale epidemic have been numerous. These include: (i) Immediately 
7. Hollensead SC, Lockwood WB, Elin RJ (2004). Errors in pathology and laboratory medicine: consequences and prevention. J Surg Oncol, 88(3): 161-181.

8. Plebani M (2009). Exploring the iceberg of errors in laboratory medicine. Clin Chim Acta, 404(1): 16-23.

9. Plebani M, Piva E (2010). Medical errors: pre-analytical issue in patient safety. J Med Biochem, 29(4): 310-314.

10. Plebani M, Carraro P (1997). Mistakes in a stat laboratory: types and frequency. Clin Chem, 43(8):1348-1351.

11. Kaushik N, Green S (2014). Pre-analytical errors: their impact and how to minimize them. MLO Med Lab Obs, 46(5): 22-24.

12. Rin G (2010). Pre-analytical workstations as a tool for reducing laboratory errors. J Med Biochem, 29(4):315-324.

13. Makitalo O, Liikanen E (2013). Improving quality at the preanalytical phase of blood sampling: literature review. Int J Biomed Lab Sci, 2:7-16.

14. Côte d'Ivoire (2020). Plan de riposte contre les infections respiratoires aiguës a coronavirus- covid-19 cote d'ivoire [Internet]. Convention de la société civile ivoirienne (csci). [Cited 2020 Nov 6]. Available from: http://csci. group/content/plan-de-riposte-contre-les-infections-respiratoires-aigu $\% \mathrm{C} 3 \% \mathrm{ABs}$-coronavirus-covid-19-cote-d

Copyright: (02021 Maxime Kouao DIANE. This is an open-access article distributed under the terms of the Creative Commons Attribution License, which permits unrestricted use, distribution, and reproduction in anymedium, provided the original author and source are credited. 